\title{
Analytical Solution for the Differential Equation Containing Generalized Fractional Derivative Operators and Mittag-Leffler-Type Function
}

\author{
V. B. L. Chaurasia and Ravi Shanker Dubey \\ Department of Mathematics, University of Rajasthan, Jaipur 302004, India \\ Correspondence should be addressed to Ravi Shanker Dubey, ravishankerdubey@indiatimes.com \\ Received 26 March 2011; Accepted 10 May 2011 \\ Academic Editor: M. F. El-Sayed \\ Copyright (C) 2011 V. B. L. Chaurasia and R. S. Dubey. This is an open access article distributed \\ under the Creative Commons Attribution License, which permits unrestricted use, distribution, \\ and reproduction in any medium, provided the original work is properly cited. \\ We discuss and derive the analytical solution for the fractional partial differential equation with \\ generalized Riemann-Liouville fractional operator $D_{0, t}^{\alpha, \beta}$ of order $\alpha$ and $\beta$. Here, we derive the \\ solution of the given differential equation with the help of Laplace and Hankel transform in terms \\ of Fox's $H$-function as well as in terms of Fox-Wright function $p \psi_{q}$.
}

\section{Introduction, Definition, and Preliminaries}

Applications of fractional calculus require fractional derivatives of different kinds [19]. Differentiation and integration of fractional order are traditionally defined by the right-sided Riemann-Liouville fractional integral operator $I_{a+}^{P}$ and the left-sided RiemannLiouville fractional integral operator $I_{a-}^{P}$, and the corresponding Riemann-Liouville fractional derivative operators $D_{a+}^{P}$ and $D_{a-}^{P}$, as follows [10, 11]:

$$
\begin{gathered}
\left(I_{a+}^{\mu} f\right)(x)=\frac{1}{\Gamma(\mu)} \int_{a}^{x} \frac{f(t)}{(x-t)^{1-\mu}} d t \quad(x>a ; R(\mu)>0), \\
\left(I_{a-}^{\mu} f\right)(x)=\frac{1}{\Gamma(\mu)} \int_{x}^{a} \frac{f(t)}{(t-x)^{1-\mu}} d t \quad(x<a ; R(\mu)>0), \\
\left(D_{a \pm}^{\mu} f\right)(x)=\left( \pm \frac{d}{d x}\right)^{n}\left(I_{a \pm}^{n-\mu} f\right)(x) \quad(R(\mu) \geq 0 ; n=[R(\mu)]+1),
\end{gathered}
$$


where the function $f$ is locally integrable, $R(\mu)$ denotes the real part of the complex number $\mu \in C$ and $[R(\mu)]$ means the greatest integer in $R(\mu)$.

Recently, a remarkable large family of generalized Riemann-Liouville fractional derivatives of order $\alpha(0<\alpha<1)$ and type $\beta(0 \leq \beta \leq 1)$ was introduced as follows [1$3,5,6,8]$.

Definition 1.1. The right-sided fractional derivative $D_{a+}^{\alpha, \beta}$ and the left-sided fractional derivative $D_{a-}^{\alpha, \beta}$ of order $\alpha(0<\alpha<1)$ and type $\beta(0 \leq \beta \leq 1)$ with respect to $x$ are defined by

$$
\left(D_{a \pm}^{\alpha, \beta} f\right)(x)=\left( \pm I_{a \pm}^{\beta(1-\alpha)} \frac{d}{d x}\left(I_{a \pm}^{(1-\beta)(1-\alpha)} f\right)\right)(x)
$$

whenever the second number of (1.4) exists. This generalization (1.4) yields the classical Riemann-Liouville fractional derivative operator when $\beta=0$. Moreover, for $\beta=1$, it gives the fractional derivative operator introduced by Liouville [12] which is often attributed to Caputo now-a-days and which should more appropriately be referred to as the LiouvilleCaputo fractional derivative. Several authors $[7,9]$ called the general operators in (1.4) the Hilfer fractional derivative operators. Applications of $D_{a \pm}^{\alpha, \beta}$ are given [3].

Using the formulas (1.1) and (1.2) in conjunction with (1.3) when $n=1$, the fractional derivative operator $D_{a \pm}^{\alpha, \beta}$ can be written in the following form:

$$
\left(D_{a \pm}^{\alpha, \beta} f\right)(x)=\left( \pm I_{a \pm}^{\beta(1-\alpha)}\left(D_{a \pm}^{\alpha+\beta-\alpha \beta} f\right)\right)(x)
$$

The difference between fractional derivatives of different types becomes apparent from their Laplace transformations. For example, it is found for $0<\alpha<1$ that $[1,2,9]$

$$
L\left[\left(D_{0+}^{\alpha, \beta} f\right)(x)\right](s)=s^{\alpha} L[f(x)](s)-s^{\beta(\alpha-1)}\left(I_{0+}^{(1-\beta)(1-\alpha)} f\right)(0+) \quad(0<\alpha<1)
$$

where $\left(I_{0+}^{(1-\beta)(1-\alpha)} f\right)(0+)$ is the Riemann-Liouville fractional integral of order $(1-\beta)(1-\alpha)$ evaluated in the limit as $t \rightarrow 0+$, it being understood (as usual) that [13],

$$
L[f(x)](s):=\int_{0}^{\infty} e^{-s x} f(x) d x:=F(s)
$$

provided that the defining integral in (1.7) exists.

The familiar Mittag-Leffler functions $E_{\mu}(z)$ and $E_{\mu, v}(z)$ are defined by the following series:

$$
\begin{aligned}
E_{\mu}(z) & :=\sum_{n=0}^{\infty} \frac{z^{n}}{\Gamma(\mu n+1)}:=E_{\mu, 1}(z) \quad(z \in C ; R(\mu)>0), \\
E_{\mu, v}(z) & :=\sum_{n=0}^{\infty} \frac{z^{n}}{\Gamma(\mu n+v)} \quad(z, v \in C ; R(\mu)>0),
\end{aligned}
$$


respectively. These functions are natural extensions of the exponential, hyperbolic, and trigonometric functions, since

$$
\begin{gathered}
E_{1}(z)=e^{z}, E_{2}\left(z^{2}\right)=\cosh z, E_{2}\left(-z^{2}\right)=\cos z \\
E_{1,2}(z)=\frac{e^{z}-1}{z}, E_{2,2}\left(z^{2}\right)=\frac{\sinh z}{z}
\end{gathered}
$$

For a detailed account of the various properties, generalizations, and applications of the Mittag-Leffler functions, the reader may refer to the recent works by, for example, Gorenflo et al. [14] and Kilbas et al. [15-17]. The Mittag-Leffler function (1.1) and some of its various generalizations have only recently been calculated numerically in the whole complex plane $[18,19]$. By means of the series representation, a generalization of the Mittag-Leffler function $E_{\mu, v}(z)$ of (1.2) was introduced by Prabhakar [20] as follows:

$$
E_{\mu, v}^{\lambda}(z)=\sum_{n=0}^{\infty} \frac{(\lambda)_{n}}{\Gamma(\mu n+v)} \frac{z^{n}}{n !} \quad(z, v, \lambda \in C ; R(\mu)>0),
$$

where $(\lambda)_{v}$ denotes the familiar Pochhammer symbol, defined (for $\lambda, v \in C$ and in terms of the familiar Gamma function) by

$$
(\lambda)_{v}:=\frac{\Gamma(\lambda+v)}{\Gamma(\lambda)}= \begin{cases}1 & (v=0 ; \lambda \in C \backslash\{0\}) \\ \lambda(\lambda+1) \cdots(\lambda+n-1) & (v=n \in N ; \lambda \in C) .\end{cases}
$$

Clearly, we have the following special cases:

$$
E_{\mu, v}^{1}(z)=E_{\mu, \nu}(z), \quad E_{\mu, 1}^{1}(z)=E_{\mu}(z)
$$

Indeed, as already observed earlier by Srivastava and Saxena [21], the generalized MittagLeffler function $E_{\mu, v}^{\lambda}(z)$ itself is actually a very specialized case of a rather extensively investigated function $p \Psi_{q}$ as indicated below [17]:

$$
E_{u, v}^{\lambda}(z)=\frac{1}{\Gamma(\lambda)} \Psi_{1}\left[\begin{array}{l}
(\lambda, 1) ; \\
(v, \mathrm{u}) ;
\end{array}\right]
$$

Here and in what follows, $p \Psi_{q}$ denotes the Wright (or more appropriately, the Fox-Wright) generalized of the hypergeometric ${ }_{p} \mathrm{~F}_{q}$ function, which is defined as follows [12]:

$$
\begin{gathered}
{ }_{p} \Psi_{q}=\left[\begin{array}{c}
\left(a_{1}, A_{1}\right), \ldots,\left(a_{p}, A_{p}\right) ; \\
\left(b_{1}, B_{1}\right), \ldots,\left(b_{q}, B_{q}\right) ;
\end{array}\right]=\sum_{x=0}^{\infty} \frac{\Gamma\left(a_{1}+A_{1} k\right) \cdots \Gamma\left(a_{p}+A_{p} k\right) z^{k}}{\Gamma\left(b_{1}+B_{1} k\right) \cdots \Gamma\left(b_{q}+B_{q} k\right) k !}, \\
{\left[R\left(A_{j}\right)>0(j=1, \ldots, p) ; R\left(B_{j}\right)>0(j=1, \ldots, q) ; 1+R\left(\sum_{j=1}^{q} B_{j}-\sum_{j=1}^{p} A_{j}\right) \geq 0\right],}
\end{gathered}
$$


in which we assumed in general that

$$
a_{j}, A_{j} \in C(j=1, \ldots, p), \quad b_{j}, B_{j} \in C(j=1, \ldots, q) .
$$

In application of Mittag-Leffler function, it is useful to have the following Laplace inverse transform formula:

$$
L^{-1}\left\{\frac{S^{\gamma-\beta}}{\left(S^{\gamma}+A\right)^{k+1}}\right\}=\frac{1}{k !} t^{\gamma k+\beta-1} E_{\gamma, \beta}^{k}\left(-A t^{\gamma}\right),
$$

where $E_{\gamma, \beta}^{j}(z)=\left(d^{j} / d z^{j}\right) E_{\gamma, \beta}(z)$.

\section{Fox's $H$-function}

The Fox function, also referred as the Fox's $H$-function, generalizes the Mellin-Barnes function. The importance of the Fox function lies in the fact that it includes nearly all special functions occurring in applied mathematics and statistics as special cases. Fox $H$-function is defined as [22]

$$
H_{p, q+1}^{1, p}\left[-x \mid \begin{array}{c}
\left(1-a_{1}, A_{1}\right), \ldots,\left(1-a_{p}, A_{p}\right) \\
(0,1),\left(1-b_{1}, B_{1}\right), \ldots,\left(1-b_{q}, B_{q}\right)
\end{array}\right]=\sum_{k=0}^{\infty} \frac{\Gamma\left(a_{1}+A_{1} k\right) \cdots \Gamma\left(a_{p}+A_{p} k\right)}{k ! \Gamma\left(b_{1}+B_{1} k\right) \cdots \Gamma\left(b_{p}+B_{q} k\right)} x^{k}
$$

We need this relation

$$
\begin{aligned}
E_{\alpha, \beta}^{k}(x) & =\sum_{n=k}^{\infty} \frac{n ! x^{n-k}}{(n-k) ! \Gamma(\alpha n+\beta)}=\sum_{j=0}^{\infty} \frac{\Gamma(j+k+1) x^{j}}{j ! \Gamma(\alpha j+\alpha k+\beta)} \\
& =H_{1,2}^{1,1}\left[-x \mid \begin{array}{c}
(-k, 1) \\
(0,1),(1-\alpha k-\beta, \alpha)
\end{array}\right] .
\end{aligned}
$$

\section{Finite Hankel Transform}

If $f(r)$ satisfies Dirichlet conditions in closed interval $(0, a)$ and if its finite Hankel transform is defined to be [23]

$$
H[f(r)]=\bar{f}\left(\lambda_{n}\right)=\int_{0}^{a} r f(r) J_{0}\left(r \lambda_{n}\right) d r
$$

where $\lambda_{n}$ are the roots of the equation $J_{0}(r)=0$. Then at each point of the interval at which $f(r)$ is continuous:

$$
f(r)=\frac{2}{a^{2}} \sum_{n=1}^{\infty} \bar{f}\left(\lambda_{n}\right) \frac{J_{0}\left(\lambda_{n} r\right)}{J_{1}^{2}\left(\lambda_{n} a\right)}
$$


where the sum is taken over all positive roots of $J_{0}(r)=0, J_{0}$ and $J_{1}$ are Bessel functions of first kind.

In application of the finite Hankel transform to physical problems, it is useful to have the following formula [23]

$$
H\left[\frac{d^{2} f}{d r^{2}}+\frac{1}{r} \frac{d f}{d r}\right]=-\lambda_{n}^{2} \bar{f}(r)+a \lambda_{n} f(a) J_{1}\left(\lambda_{n} a\right)
$$

Example 3.1. Solve the differential equation

$$
D_{0, t}^{2 \alpha, \beta} u(r, t)+a D_{0, t}^{\alpha, \beta} u(r, t)=d\left(\frac{\partial^{2} u(r, t)}{\partial r^{2}}+\frac{1}{r} u(r, t)\right)+f(t)
$$

where $0<\alpha \leq 1 / 2$ and $0 \leq \beta \leq 1$ with initial condition

$$
\begin{gathered}
I_{t}^{(1-\beta)(1-2 \alpha)} u(r, 0)=\phi_{1}(r), \\
I_{t}^{(1-\beta)(1-\alpha)} u(r, 0)=\phi_{2}(r), \\
u(r, t)=0 \text { everywhere for } t<0, \\
u(r, t)=0 \text { for } r=1, t>0, \\
u(r, t)=\text { finite at } r=0, t>0 .
\end{gathered}
$$

Solution 1. Taking Laplace transform of (3.4), we get

$$
s^{2 \alpha} \widetilde{u}(r, s)-s^{\beta(2 \alpha-1)} \phi_{1}(r)+a s^{\alpha} \tilde{u}(r, s)-a s^{\beta(\alpha-1)} \phi_{2}(r)=d\left[\frac{\partial^{2} \widetilde{u}(r, s)}{\partial r^{2}}+\frac{1}{r} \widetilde{u}(r, s)\right]+\tilde{f}(s) .
$$

Taking Hankel transform on both side of the above equation, we get

$$
\begin{aligned}
& s^{2 \alpha} \tilde{\widetilde{u}}(r, s)-s^{\beta(2 \alpha-1)} \tilde{\phi}_{1}(r)+a s^{\alpha} \tilde{\widetilde{u}}(r, s)-a s^{\beta(\alpha-1)} \tilde{\phi}_{2}(r) \\
& \quad=d\left[-\lambda_{n}^{2} \tilde{\widetilde{u}}(r, s)\right]+\tilde{f}(s) \frac{J_{1}\left(\lambda_{n}\right)}{\lambda_{n}},
\end{aligned}
$$


then we get

$$
\begin{gathered}
\tilde{\widetilde{u}}(r, s)=\frac{s^{\beta(2 \alpha-1)} \tilde{\phi}_{1}(r)}{\left(s^{2 \alpha}+a s^{\alpha}+d \lambda_{n}^{2}\right)}+\frac{a s^{\beta(\alpha-1)} \tilde{\phi}_{2}(r)}{\left(s^{2 \alpha}+a s^{\alpha}+d \lambda_{n}^{2}\right)}+\frac{\tilde{f}(s)}{\left(s^{2 \alpha}+a s^{\alpha}+d \lambda_{n}^{2}\right)} \frac{J_{1}\left(\lambda_{n}\right)}{\lambda_{n}}, \\
\tilde{\widetilde{u}}(r, s)=\widetilde{\widetilde{G}}_{1} \tilde{\phi}_{1}(r)+a \widetilde{\widetilde{G}}_{2} \tilde{\phi}_{2}(r)+\widetilde{\widetilde{G}}_{3} \tilde{f}(s) \frac{J_{1}\left(\lambda_{n}\right)}{\lambda_{n}}
\end{gathered}
$$

where

$$
\begin{aligned}
& \widetilde{\widetilde{G}}_{1}=\frac{s^{\beta(2 \alpha-1)}}{\left(s^{2 \alpha}+a s^{\alpha}+d \lambda_{n}^{2}\right)}, \\
& \widetilde{\widetilde{G}}_{2}=\frac{s^{\beta(\alpha-1)}}{\left(s^{2 \alpha}+a s^{\alpha}+d \lambda_{n}^{2}\right)}, \\
& \widetilde{\widetilde{G}}_{3}=\frac{1}{\left(s^{2 \alpha}+a s^{\alpha}+d \lambda_{n}^{2}\right)} .
\end{aligned}
$$

On taking Laplace inverse of (3.10), (3.11), and (3.12), respectively,

$$
\begin{gathered}
L^{-1}\left\{\frac{s^{\beta(2 \alpha-1)}}{\left(s^{2 \alpha}+a s^{\alpha}+d \lambda_{n}^{2}\right)}\right\}=\sum_{m=0}^{\infty} \frac{(-1)^{m}}{a^{m+1}} \frac{t^{\alpha+\beta-2 \alpha \beta-m \alpha-1}}{m !} E_{\alpha, \alpha+\beta-2 \alpha \beta-2 m \alpha}^{m}\left(\frac{-d \lambda_{n}^{2}}{a} t^{\alpha}\right), \\
L^{-1}\left\{\frac{s^{\beta(\alpha-1)}}{\left(s^{2 \alpha}+a s^{\alpha}+d \lambda_{n}^{2}\right)}\right\}=\sum_{m=0}^{\infty} \frac{(-1)^{m}}{a^{m+1}} \frac{t^{\alpha+\beta-\alpha \beta-m \alpha-1}}{m !} E_{\alpha, \alpha+\beta-\alpha \beta-2 m \alpha}^{m}\left(\frac{-d \lambda_{n}^{2}}{a} t^{\alpha}\right), \\
L^{-1}\left\{\frac{1}{\left(s^{2 \alpha}+a s^{\alpha}+d \lambda_{n}^{2}\right)}\right\}=\sum_{m=0}^{\infty} \frac{(-1)^{m}}{a^{m+1}} \frac{t^{\alpha-m \alpha-1}}{m !} E_{\alpha, \alpha-2 m \alpha}^{m}\left(\frac{-d \lambda_{n}^{2}}{a} t^{\alpha}\right) .
\end{gathered}
$$

After taking Inverse Laplace and Hankel transform of (3.9) put the value (3.13) through (3.15) in (3.9), we get

$$
\begin{aligned}
u(r, t)= & 2 \sum_{n=0}^{\infty} \sum_{m=0}^{\infty} \frac{(-1)^{m}}{a^{m+1}} \frac{J_{0}\left(\lambda_{n} r\right)}{m ! J_{1}^{2}\left(\lambda_{n}\right)} \phi_{1}(r) t^{-2 \alpha \beta-m \alpha+\alpha+\beta-1} \sum_{j=0}^{\infty} \frac{(j+m+1) !\left(-d \lambda_{n}^{2} t^{\alpha} / a\right)^{j}}{j ! \Gamma(j \alpha+\alpha+\beta-2 \alpha \beta-2 m \alpha)} \\
& +2 a \sum_{n=0}^{\infty} \sum_{m=0}^{\infty} \frac{(-1)^{m}}{a^{m+1}} \frac{J_{0}\left(\lambda_{n} r\right)}{J_{1}^{2}\left(\lambda_{n}\right)} \phi_{2}(r) t^{-\alpha \beta-m \alpha+\alpha+\beta-1} \sum_{j=0}^{\infty} \frac{(j+m+1) !\left(-d \lambda_{n}^{2} t^{\alpha} / a\right)^{j}}{j ! \Gamma(j \alpha+\alpha+\beta-\alpha \beta-m \alpha)} \\
& +2 \sum_{n=0}^{\infty} \sum_{m=0}^{\infty} \frac{(-1)^{m}}{a^{m+1}} \frac{J_{0}\left(\lambda_{n} r\right)}{\lambda_{n} J_{1}\left(\lambda_{n}\right)} \int_{0}^{t} u^{\alpha-m \alpha-1} \sum_{j=0}^{\infty} \frac{(j+m+1) !\left(-d \lambda_{n}^{2} u^{\alpha} / a\right)^{j}}{j ! \Gamma(\alpha j+\alpha-m \alpha)} f(t-u) d u .
\end{aligned}
$$




$$
\begin{aligned}
u(r, t)= & 2 \sum_{n=0}^{\infty} \sum_{m=0}^{\infty} \frac{(-1)^{m}}{a^{m+1}} \frac{J_{0}\left(\lambda_{n} r\right)}{J_{1}^{2}\left(\lambda_{n}\right)} \phi_{1}(r) t^{-2 \alpha \beta-m \alpha+\alpha+\beta-1} \\
& \cdot H_{1,2}^{1,1}\left[\frac{d \lambda_{n}^{2} t^{\alpha}}{a} \mid \begin{array}{c}
(-m-1,1) \\
(0,1),(1-\alpha-\beta+2 \alpha \beta+2 m \alpha, \alpha)
\end{array}\right] \\
& +2 a \sum_{n=0}^{\infty} \sum_{m=0}^{\infty} \frac{(-1)^{m}}{a^{m+1}} \frac{J_{0}\left(\lambda_{n} r\right)}{J_{1}^{2}\left(\lambda_{n}\right)} \phi_{2}(r) t^{-\alpha \beta-m \alpha+\alpha+\beta-1} \\
& \cdot H_{1,2}^{1,1}\left[\frac{d \lambda_{n}^{2} t^{\alpha}}{a} \mid \begin{array}{cc}
(-m-1,1) \\
(0,1),(1-\alpha-\beta+\alpha \beta+m \alpha, \alpha)
\end{array}\right] \\
& +2 \sum_{n=0}^{\infty} \sum_{m=0}^{\infty}(-1)^{n} \frac{J_{0}\left(\lambda_{n} r\right)}{\lambda_{n} J_{1}\left(\lambda_{n}\right)} \int_{0}^{t} u^{\alpha-m \alpha-1} H_{1,2}^{1,1}\left[\frac{d \lambda_{n}^{2} u^{\alpha}}{a} \mid \begin{array}{c}
(-m-1),(1-\alpha+m \alpha, \alpha)
\end{array}\right] f(t-u) d u .
\end{aligned}
$$

Example 3.2. Solve the differential equation (3.4) with initial condition

$$
\begin{gathered}
I_{t}^{(1-\beta)(1-2 \alpha)} u(r, 0)=0, \\
I_{t}^{(1-\beta)(1-\alpha)} u(r, 0)=0, \\
u(r, t)=0 \quad \text { everywhere for } t \leq 0, \\
u(r, t)=0 \text { for } r=1, t>0, \\
u(r, t)=\text { finite at } r=0, t>0 .
\end{gathered}
$$

Solution 2. Taking Laplace and Hankel transform of (3.4), we get

$$
\widetilde{\widetilde{u}}(r, s)=\frac{J_{1}\left(\lambda_{n}\right)}{\lambda_{n}} \frac{\tilde{f}(s)}{\left(s^{2 \alpha}+a s^{\alpha}+d \lambda_{n}^{2}\right)},
$$

on taking Inverse Laplace transform of equation (3.19), we get

$$
\tilde{u}(r, t)=L^{-1}\left\{\tilde{f}(s) \frac{J_{1}\left(\lambda_{n}\right)}{\lambda_{n}}\right\} L^{-1}\left\{\left(\frac{1}{s^{2 \alpha}+a s^{\alpha}+d \lambda_{n}^{2}}\right)\right\}
$$

By using convolution theorem for Laplace transform and taking inverse Hankel transform, we get

$$
u(r, t)=2 \sum_{n=0}^{\infty} \sum_{m=0}^{\infty} \frac{(-1)^{m}}{a^{m+1}} \frac{J_{0}\left(\lambda_{n} r\right)}{\lambda_{n} J_{1}\left(\lambda_{n}\right)} \int_{0}^{t} u^{\alpha-m \alpha-1} E_{\alpha, \alpha-2 m \alpha}^{m}\left(-\frac{d \lambda_{n}^{2} u^{\alpha}}{a}\right) f(t-u) d u
$$


or

$$
u(r, t)=2 \sum_{n=0}^{\infty} \sum_{m=0}^{\infty} \frac{(-1)^{m}}{a^{m+1}} \frac{J_{0}\left(\lambda_{n} r\right)}{\lambda_{n} J_{1}\left(\lambda_{n}\right)} \int_{0}^{t} u^{\alpha-m \alpha-1} \sum_{j=0}^{\infty} \frac{(j+m+1) !}{(j) !} \frac{\left(-d \lambda_{n}^{2} u^{\alpha} / a\right)^{j}}{\Gamma(\alpha j+\alpha-m \alpha)} .
$$

By using the relation (2.2)

$$
u(r, t)=2 \sum_{n=0}^{\infty} \sum_{m=0}^{\infty} \frac{(-1)^{m}}{a^{m+1}} \frac{J_{0}\left(\lambda_{n} r\right)}{\lambda_{n} J_{1}\left(\lambda_{n}\right)} \int_{0}^{t} u^{\alpha-m \alpha-1} H_{1,2}^{1,1}\left[\frac{d \lambda_{n}^{2} u^{\alpha}}{a} \mid \begin{array}{c}
(-m-1,1) \\
(0,1),(1-\alpha+m \alpha, \alpha)
\end{array}\right] f(t-u) d u,
$$

or

$$
u(r, t)=2 \sum_{n=0}^{\infty} \sum_{m=0}^{\infty} \frac{(-1)^{m}}{a^{m+1}} \frac{J_{0}\left(\lambda_{n} r\right)}{\lambda_{n} J_{1}\left(\lambda_{n}\right)} \int_{0}^{t} u^{\alpha-m \alpha-1} \frac{1}{\Gamma(m)}{ }_{1} \Psi_{1}\left[\begin{array}{c}
(m, 1) ; \\
(\alpha-2 m \alpha, \alpha) ;
\end{array}-\frac{d \lambda_{n}^{2} u^{\alpha}}{a}\right],
$$

which is the required solution.

\section{References}

[1] R. Hilfer, Applications of Fractional Calculus in Physics, World Scientific Publishing, River Edge, NJ, USA, 2000.

[2] R. Hilfer, "Fractional time evolution," in Applications of Fractional Calculus in Physics, R. Hilfer, Ed., pp. 87-130, World Scientific Publishing, River Edge, NJ, USA, 2000.

[3] R. Hilfer, "Experimental evidence for fractional time evolution in glass forming materials," Chemical Physics, vol. 284, no. 1-2, pp. 399-408, 2002.

[4] R. Hilfer, "Threefold Introduction to fractional derivatives," in Anomalous Transport: Foundations and Applications, R. Klages, G. Radons, and I. M. Sokolov, Eds., pp. 17-73, Wiley-VCH Verlag, Weinheim, Germany, 2008.

[5] R. Hilfer and L. Anton, "Fractional master equations and fractal time random walks," Physical Review E, vol. 51, no. 2, pp. R848-R851, 1995.

[6] R. Hilfer, Y. Luchko, and Ž. Tomovski, "Operational method for the solution of fractional differential equations with generalized Riemann-Liouville fractional derivatives," Fractional Calculus $\mathcal{E}$ Applied Analysis, vol. 12, no. 3, pp. 299-318, 2009.

[7] F. Mainardi and R. Gorenflo, "Time-fractional derivatives in relaxation processes: a tutorial survey," Fractional Calculus and Applied Analysis, vol. 10, no. 3, pp. 269-308, 2007.

[8] T. Sandev and Ž. Tomovski, "The general time fractional wave equation for a vibrating string," Journal of Physics. A. Mathematical and Theoretical, vol. 43, no. 5, 2010.

[9] H. M. Srivastava and Ž. Tomovski, "Fractional calculus with an integral operator containing a generalized Mittag-Leffler function in the kernel," Applied Mathematics and Computation, vol. 211, no. 1, pp. 198-210, 2009.

[10] A. Erdélyi, W. Magnus, F. Oberhettinger, and F. G. Tricomi, Tables of Integral Transforms, vol. 2, McGraw-Hill, London, UK, 1954.

[11] S. G. Samko, A. A. Kilbas, and O. I. Marichev, Fractional Integrals and Derivatives: Theory and Applications, Gordon and Breach Science Publishers, New York, NY, USA, 1993.

[12] J. Liouville, "Mémoire sur quelques quéstions de géometrie et de mécanique, et sur un nouveau genre de calcul pour résoudre ces quéstions," École Polytechnique, vol. 13, no. 21, pp. 1-69, 1832.

[13] A. Erdélyi, W. Magnus, F. Oberhettinger, and F. G. Tricomi, Tables of Integral Transforms, vol. 1, McGraw-Hill, London, UK, 1954. 
[14] R. Gorenflo, F. Mainardi, and H. M. Srivastava, "Special functions in fractional relaxation-oscillation and fractional diffusion-wave phenomena," in Proceedings of the 8th International Colloquium on Differential Equations, D. Bainov, Ed., pp. 195-202, VSP, Plovdiv, Bulgaria, August 1997.

[15] A. A. Kilbas, M. Saigo, and R. K. Saxena, "Solution of Volterra integrodifferential equations with generalized Mittag-Leffler function in the kernels," Journal of Integral Equations and Applications, vol. 14, no. 4, pp. 377-396, 2002.

[16] A. A. Kilbas, M. Saigo, and R. K. Saxena, "Generalized Mittag-Leffler function and generalized fractional calculus operators," Integral Transforms and Special Functions, vol. 15, no. 1, pp. 31-49, 2004.

[17] A. A. Kilbas, H. M. Srivastava, and J. J. Trujillo, Theory and Applications of Fractional Differential Equations, vol. 204 of North-Holland Mathematics Studies, Elsevier, Amsterdam, The Netherlands, 2006.

[18] R. Hilfer and H. Seybold, "Computation of the generalized Mittag-Leffler function and its inverse in the complex plane," Integral Transforms and Special Functions, vol. 17, no. 9, pp. 637-652, 2006.

[19] H. J. Seybold and R. Hilfer, "Numerical results for the generalized Mittag-Leffler function," Fractional Calculus and Applied Analysis, vol. 8, no. 2, pp. 127-139, 2005.

[20] T. R. Prabhakar, "A singular integral equation with a generalized Mittag Leffler function in the kernel," Yokohama Mathematical Journal, vol. 19, pp. 7-15, 1971.

[21] H. M. Srivastava and R. K. Saxena, "Some Volterra-type fractional integro-differential equations with a multivariable confluent hypergeometric function as their kernel," Journal of Integral Equations and Applications, vol. 17, no. 2, pp. 199-217, 2005.

[22] R. Metzler and J. Klafter, "The random walk's guide to anomalous diffusion: a fractional dynamics approach," Physics Reports, vol. 339, no. 1, p. 77, 2000.

[23] I. N. Sneddon, Fourier Transforms, McGraw-Hill, New York, NY, USA, 1951. 


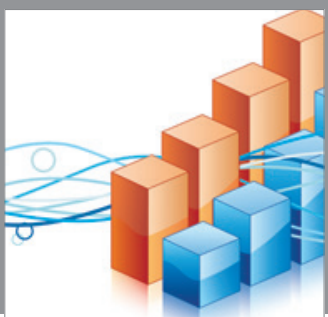

Advances in

Operations Research

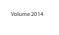

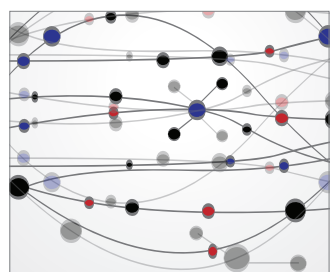

\section{The Scientific} World Journal
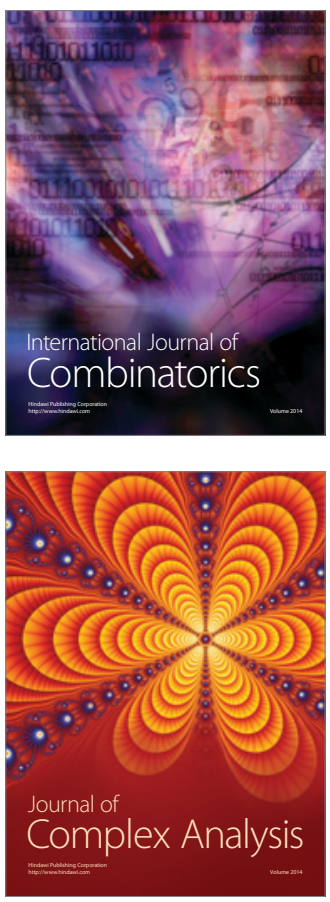

International Journal of

Mathematics and

Mathematical

Sciences
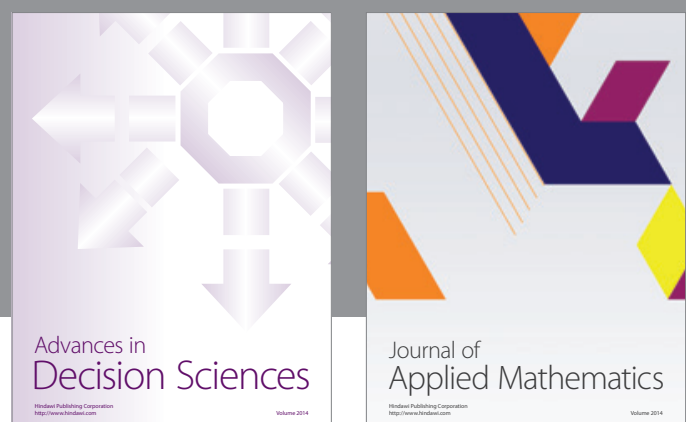

Journal of

Applied Mathematics
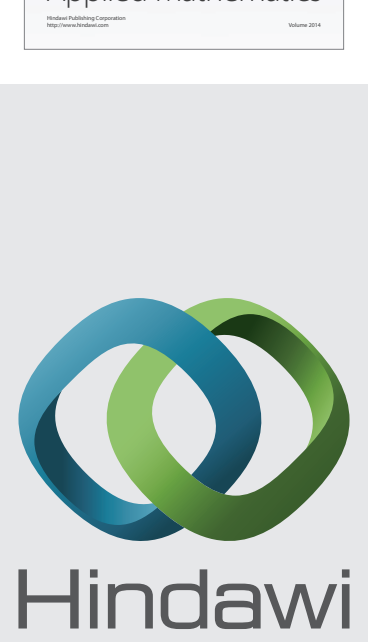

Submit your manuscripts at http://www.hindawi.com
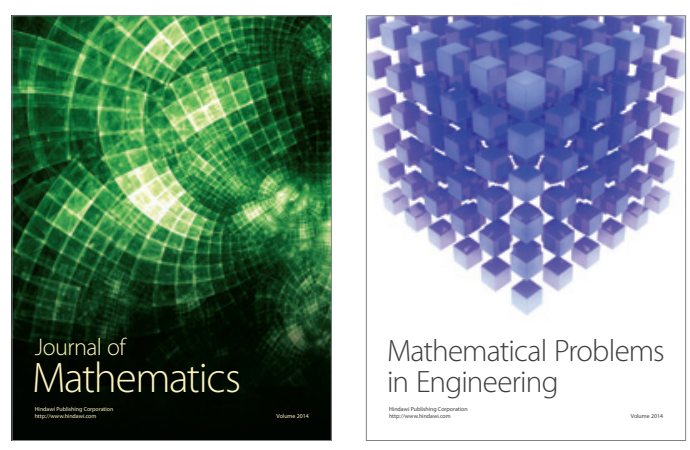

Mathematical Problems in Engineering
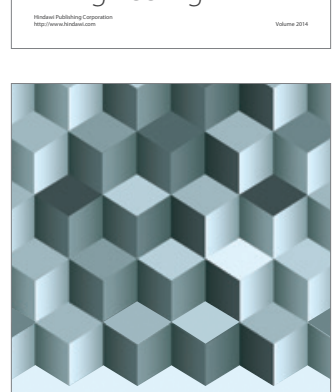

Journal of

Function Spaces
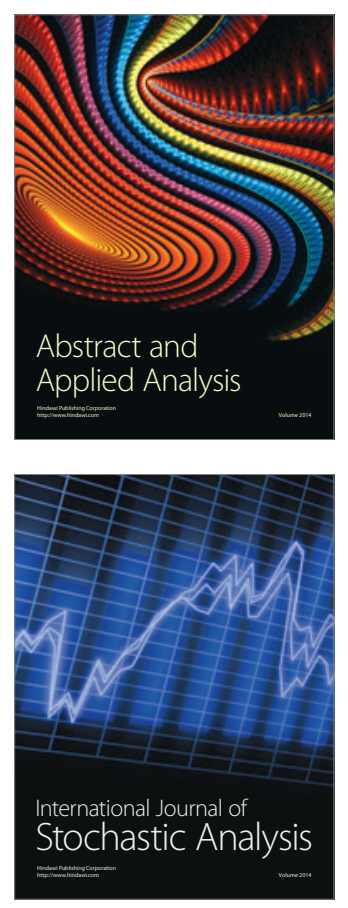

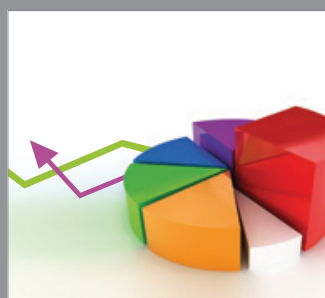

ournal of

Probability and Statistics

Promensencen
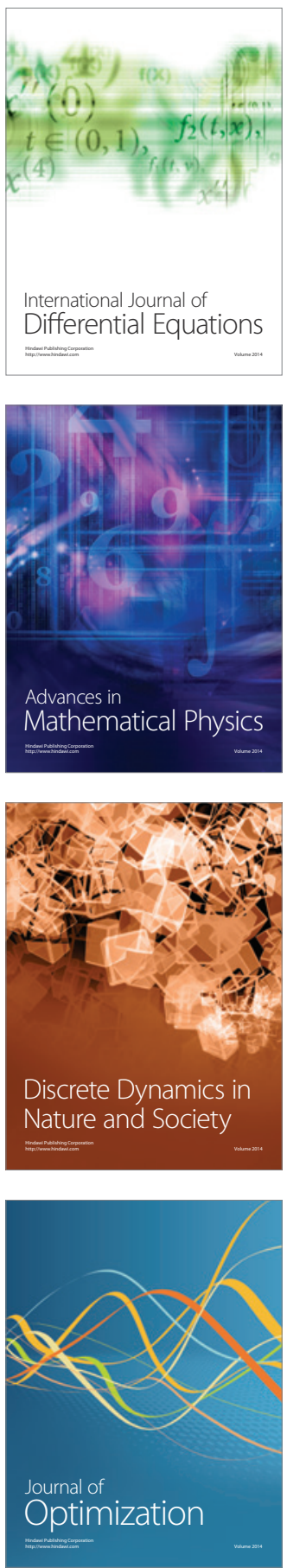